Japan. J. Math.

Vol. 22, No. 2, 1996

\title{
A remark on decay properties of solutions of nonlinear parabolic variational inequalities
}

\author{
By Haruo NAGASE
}

(Received August 19, 1994)

\section{Introduction}

Let $\Omega$ be a bounded starshaped domain in $R^{n}$ with coordinates $x=$ $\left(x_{1}, \ldots, x_{n}\right)$, its boundary $\Gamma$ be of class $C^{1}$ and $\Gamma_{1}$ be a nonempty relatively open subset of $\Gamma$.

We assume that the usual Sobolev spaces $L^{q}(\Omega)$ and $W^{1, p}(\Omega)$ are known. We denote the norms in $L^{q}(\Omega)$ and $W^{1, p}(\Omega)$ by ||$_{q}$ and ||$_{1, p}$, respectively. The space $V$ is the completion of $C_{(0)}^{\infty}(\bar{\Omega})$, the family of all infinitely differentiable functions in $\bar{\Omega}$ vanishing in each neighborhood of $\bar{\Gamma}_{1}$, with respect to the norm | $\left.\right|_{1, p}$. Throughout this paper we denote $L^{2}(\Omega)$ by $H$ and the dual space of $V$ by $V^{*}$. The norms in $V, H$ and $V^{*}$ are written by $\left.\right|_{V},||_{H}$ and $\mid V_{V^{*}}$, respectively. We denote the pairing between $V$ and $V^{*}$ by $\langle$,$\rangle , the inner product of H$ by $($, and the adjoint number of $p$ by $p^{*}$, i.e., $p^{*}=p /(p-1)$.

Let $X$ be a Banach space with the norm ||$_{X}$. Let $s$ be a real number with $1 \leqq s<\infty$, and let $I$ be an open interval in $R^{1}$. with a parameter $t$. We denote by $L^{s}(I, X)$ the space of equivalent classes of functions $v(t) ; I \rightarrow X$, which are $L^{s}$ integrable on $I$. It is a Banach space with the norm $\|v\|_{L^{s}(I, X)}=\left(\int_{I}|v(t)|_{X}^{s} d t\right)^{1 / s}$. If $s=\infty, L^{\infty}(I, X)$ means the set of all measurable functions $v(t) ; I \rightarrow X$, satisfying $\|v\|_{L^{\infty}(I, X)}=$ ess. sup. $|v(s)|_{X}<\infty$. Further, for any $s, 1 \leqq s \leqq \infty$, let us denote by $L_{\text {loc }}^{s}(I, X)$ the set of all functions $v(t) ; I \rightarrow X$, such that $v \in L^{s}(J, X)$ for any bounded open interval $J$ with $\bar{J} \subset I$. Let $C(I, X)$ be the space of all functions $v(t) ; I \rightarrow X$, which are continuous on $I$. A function $v(t) ; I \rightarrow X$ is said to be strongly differentiable at $t \in I$ if there exists $w$ in $X$ such that $\lim _{h \rightarrow 0} \mid(v(t+h)-$ $v(t)) / h-\left.w\right|_{X}=0$. And $w$ is called the strong derivative of $v(t)$ at $t \in I$. We denote the strong derivative of $v(t)$ by $v_{t}(t)$. The derivative of $v(t)$ (resp. $v(x, t)$ ) in the sense of distributions in $I$ (resp. $\Omega \times I$ ) with respect to $t$ is denoted by $(d / d t) v(t)$ (resp. $\left.D_{t} v(x, t)\right)$.

In this paper we assume that $1<p<2$ if $n=1,2$ and $2 n /(n+2)<p<2$ if $3 \leqq n$. 
We define an operator $L_{p} ; V \rightarrow V^{*}$ as follows:

$$
\left\langle L_{p} v, w\right\rangle=\sum_{j=1}^{n}\left(|\nabla v|^{p-2} v_{x_{j}}, w_{x_{j}}\right), \quad v, w \in V
$$

where $\nabla v=\left(v_{x_{1}}, \ldots, v_{x_{n}}\right)$ and $v_{x_{j}}=\left(\partial v / \partial x_{j}\right), j=1, \ldots, n$.

Let us assume that $f(t) \in V^{*}$ for any $t \in R_{+}^{1}, f_{t} \in L_{\text {loc }}^{2}\left(R_{+}^{1}, H\right)$ and $u_{0} \in V$, where $R_{+}^{1}=(0, \infty)$. We denote by $K$ a closed convex subset in $V$. We assume that $0 \in K$. We consider the nonlinear parabolic variational inequality

$$
\left\{\begin{array}{l}
u(t) \in K \text { for all } t \in R_{+}^{1} \\
\left(u_{t}(t), u(t)-v\right)+\left\langle L_{p} u(t), u(t)-v\right\rangle \leqq\langle f(t), u(t)-v\rangle \\
\quad \text { for any } v \in K \text { a.e. } t \in R_{+}^{1} \\
u(x, 0)=u_{0}(x)
\end{array}\right.
$$

where it is assumed that $u \in L_{\mathrm{loc}}^{\infty}\left(R_{+}^{1}, V\right) \cap C\left(R_{+}^{1}, H\right)$ and $u_{t} \in L_{\mathrm{loc}}^{\infty}\left(R_{+}^{1}, H\right)$.

In this paper we will show the decay estimate at infinity $(t=\infty)$ for solutions of (1.1).

We have already proved the unique existence of solutions of (1.1) in [N1] and established some asymptotic properties for solutions of (1.1) in [N2] when we replace the operator $L_{p}$ in (1.1) by a general nonlinear degenerate operator $A(t) ; V \rightarrow V^{*}$. In [N1] and [N2] we assumed that $2<p$. However, we can show the same results as in [N1] and [N2] with slight modifications under the conditions on $p$ in this paper. Especially, if we replace the operator $L_{p}$ in (1.1) by either the operator $L_{p}+c$ for a positive constant $c$ or the operator $L_{p}+L_{2}$, all of the decay properties in [N2] are valid. But we cannot apply this to our case.

From now on we denote simply $F(t)=O\left(t^{-\alpha}\right), 0<\alpha\left(\right.$ resp. $\left.O\left(e^{-\kappa t}\right), 0<\kappa\right)$ when $F(t)=O\left(t^{-\alpha}\right)\left(\operatorname{resp} . O\left(e^{-\kappa t}\right)\right)$ at $t=\infty$.

Our theorem is as follows:

Theorem. Let $\int_{0}^{\infty}|f(s)|_{H}^{2} d s<\infty$. Then the following decay properties at $t=\infty$ hold for any solution $u(t)$ of $(1.1)$ :

(I) If $\int_{t}^{t+1}\left|f_{t}(s)\right|_{H}^{2} d s=O\left(t^{-\beta_{1}}\right)$ and $\int_{t}^{t+1}|f(s)|_{V^{*}}^{p^{*}} d s=O\left(t^{-\beta_{2}}\right), \beta_{1}, \beta_{2}>$ 0 , then $|u(t)|_{H}^{2}=O\left(t^{-\beta_{2}}\right), \quad\left|u_{t}(t)\right|_{H}^{2}=O\left(t^{-\beta_{1}}\right), \int_{t}^{t+1} \int_{\Omega}\left|D_{t} \nabla u\right|^{p} d x d s=$ $O\left(t^{-\left\{\beta_{2}+\left(\beta_{1}-\beta_{2}\right) p / 2\right\}}\right)$ and $|\nabla u(t)|_{p}=O\left(t^{-\left(\beta_{1}+\beta_{2}\right) /(2 p)}\right)$ if $\beta_{1}<\beta_{2},|\nabla u(t)|_{p}=$ $O\left(t^{-\beta_{2} / p}\right)$ if $\beta_{1}>\beta_{2}$.

(II) If $\int_{t}^{t+1}\left|f_{t}(s)\right|_{H}^{2} d s=O\left(e^{-\omega t}\right)$ and $\int_{t}^{t+1}|f(s)|_{V^{*}}^{p^{*}} d s=O\left(e^{-\omega t}\right), \omega>0$, then $|u(t)|_{H}^{2}=O\left(e^{-\mu t}\right),\left|u_{t}(t)\right|_{H}^{2}=O\left(e^{-\mu t}\right), \int_{t}^{t+1} \int_{\Omega}\left|D_{t} \nabla u\right|^{p} d x d s=O\left(e^{-\mu t}\right)$ and $|\nabla u(t)|_{p}=O\left(e^{-\mu t}\right)$ for some $\mu>0$.

N.M. Bokalo investigated the following nonlinear parabolic equation of higher order: $u_{t}+(-1)^{m} \sum_{|\alpha| \leqq m} D^{\alpha}\left(\left|D^{\alpha} u\right|^{p-2} D^{\alpha} u\right)=f$ in $\Omega$ and $D^{\delta} u=0$ on $\Gamma$ for $\delta$, 
$|\delta| \leqq m-1$, when $2 \leqq p$. He proved some stability properties of $|u(t)|_{W^{m, p}(\Omega)}$ and $|u(t)|_{H}$ for any solution $u(t)$. M. Chipot-J.F. Rodrigues considered the nonlinear parabolic variational inequality containing our problem (1.1) when $1<p<$ $\infty$. They proved the decay estimate for any solution $u(t)$ of such problem in $L^{1}(\Omega)$. Namely, they showed that $|u(t)|_{1}=O\left(t^{-\alpha}\right)$ if $\int_{t}^{t+1}|f(s)|_{1} d s=O\left(t^{-\alpha}\right)$, and $|u(t)|_{1}=O\left(e^{-\kappa t}\right)$ if $\int_{t}^{t+1}|f(s)|_{1} d s=O\left(e^{-\kappa t}\right)$, where $\alpha$ and $\kappa$ are some positive numbers. G.M. Ghidaglia-A. Marzocchi obtained the exact decay estimate for $|u(t)|_{H}$ of any solution $u(t)$ for the following nonlinear parabolic equation under the assumption that $2<p: u_{t}-\operatorname{div}\left(|\nabla u|^{p-2} \nabla u\right)=0$ in $\Omega \times R_{+}^{1}, u(x, t)=0$ on $\Gamma \times R_{+}^{1}$ and $u(x, 0)=u_{0}(x)$ in $\Omega$. More precisely, they proved that $|u(t)|_{H}=O\left(t^{-1 /(p-2)}\right)$. We refer to [N2] for other results concerning with our problem and detailed references.

In Section 2 we prepare some lemmas in order to prove our theorem. In Section 3 we prove our theorem. In the following $C$ and $c$ denote positive constants which may change from line to line.

\section{Lemmas}

LEMma 2.1 (c.f., e.g., [T]). There exists a positive constant $c_{0}$ such that the inequality

$$
\sum_{j=1}^{n}\left(|\eta|^{p-2} \eta_{j}-\left|\eta^{\prime}\right|^{p-2} \eta_{j}^{\prime}\right)\left(\eta_{j}-\eta_{j}^{\prime}\right) \geqq c_{0}\left(|\eta|+\left|\eta^{\prime}\right|\right)^{p-2}\left|\eta-\eta^{\prime}\right|^{2}
$$

holds for any $\eta, \eta^{\prime} \in R^{n}$ with $|\eta|+\left|\eta^{\prime}\right| \neq 0$.

Next, let us consider the ordinary differential inequality

$$
y^{\prime}(t)+k y(t) \leqq g(t) \text { a.e. } t>0, \quad y(0) \geqq 0,
$$

where $k>0, y(t)$ is absolutely continuous on $R_{+}^{1}$ and $y^{\prime}(t)$ is the derivative in the usual sense. We assume that $g(t)$ is non-negative on $R_{+}^{1}$ and integrable on $(0, T)$ for any $T>0$. As for the asymptotic property of any solution $y(t)$ of $(2.2)$ we have the following

LEMMA 2.2 ([Ro]).

(I) If $\int_{t}^{t+1} g(s) d s=O\left(t^{-\beta}\right), \beta>0$, then $y(t)=O\left(t^{-\beta}\right)$.

(II) If $\int_{t}^{t+1} g(s) d s=O\left(e^{-\kappa t}\right), \kappa>0$, then $y(t)=O\left(e^{-\nu t}\right)$, for a positive constant $\nu$ which depends on $k$ and $\kappa$ only.

We give a lemma of Sobolev's type.

LEMMA 2.3 (Lemma 2.3 in [N2]). Let $I$ be a bounded open interval in $R^{1}$ and $z(t)$ be a function in $L^{p}(I)$ such that $(d / d t) z(t) \in L^{p}(I)$. Then for any $\varepsilon>0$ 
the estimate

$$
\|z\|_{L^{\infty}(I)} \leqq C\left(1+\varepsilon^{-\left(p^{*}-1\right)}\right)\|z\|_{L^{p}(I)}+\varepsilon\|(d / d t) z\|_{L^{p}(I)}
$$

holds, where $C$ does not depend on $\varepsilon$ and $z$.

Lemma 2.4 (Lemma 2.6 in [N2]). Let $I$ be a bounded open interval in $R^{1}$. If $v$ and $D_{t} v$ belong to $L^{q}(\Omega \times I), 1<q<\infty$, then $(d / d t)|v(t)|_{q}$ belongs to $L^{q}(I)$ and the inequality

$$
\left.\left.\int_{I}|(d / d t)| v(t)\right|_{q}\right|^{q} d t \leqq C \int_{\Omega \times I}\left|D_{t} v(x, t)\right|^{q} d x d t
$$

holds, where we denote by $(d / d t)$ and $D_{t}$ the differential with respect to $t$ in the sense of distributions in $I$ and $\Omega \times I$, respectively.

At last, we prepare a priori estimate in $V$ for solutions of (1.1) in the following

LEMMA 2.5 (c.f., e.g., [Bi]). If $\int_{0}^{\infty}|f(s)|_{H}^{2} d s<\infty$ and $u_{0} \in V$, then for any solution $u(t)$ of (1.1) it holds that

$$
|u(t)|_{V} \leqq M \text { uniformly in } t \in R_{+}^{1} \text {. }
$$

Here $M$ is a positive constant which depends on $f$ and $u_{0}$ only.

This lemma is well-known.

\section{Proof of our theorem}

Let us set $v=0$ in (1.1) to get the inequality

$$
\left(u_{t}(s), u(s)\right)+\left\langle L_{p} u(s), u(s)\right\rangle \leqq\langle f(s), u(s)\rangle \text { a.e. } s>0 .
$$

After applying Hölder's and Poincarè's inequalities to the right-hand side of (3.1), let us use Young's inequality. Then,

$$
\frac{d}{d s}|u(s)|_{H}^{2}+c|u(s)|_{V}^{p} \leqq C|f(s)|_{V^{*}}^{p^{*}} \text { a.e. } s>0 .
$$

Let us remark that the following estimates hold: by Lemma 2.5 and the condition of $p<2$

$$
|u(s)|_{V}^{2} \leqq C|u(s)|_{V}^{p}
$$

by Sobolev's inequality

$$
|u(s)|_{H} \leqq C|u(s)|_{V}
$$


where we have used the condition that $2 n /(n+2)<p$, i.e., $2<n p /(n-p)$. From (3.2)-(3.4) we get

$$
\frac{d}{d s}|u(s)|_{H}^{2}+c|u(s)|_{H}^{2} \leqq C|f(s)|_{V^{*}}^{p^{*}} \text { a.e. } s>0 .
$$

By (3.5) and Lemma 2.2,

$$
|u(t)|_{H}^{2}= \begin{cases}O\left(t^{-\beta_{2}}\right) & \text { if } \int_{t}^{t+1}|f(s)|_{V^{*}}^{p^{*}} d s=O\left(t^{-\beta_{2}}\right) \\ O\left(e^{-\nu_{1} t}\right) & \text { if } \int_{t}^{t+1}|f(s)|_{V^{*}}^{p^{*}} d s=O\left(e^{-\omega t}\right)\end{cases}
$$

with a positive number $\nu_{1}$.

After integrating (3.2) over $(t, t+1)$ for any positive number $t$, we use (3.6) to get

$$
\int_{t}^{t+1}|u(s)|_{V}^{p} d s= \begin{cases}O\left(t^{-\beta_{2}}\right) & \text { if } \int_{t}^{t+1}|f(s)|_{V^{*}}^{p^{*}} d s=O\left(t^{-\beta_{2}}\right) \\ O\left(e^{-\nu_{1} t}\right) & \text { if } \int_{t}^{t+1}|f(s)|_{V^{*}}^{p^{*}} d s=O\left(e^{-\omega t}\right)\end{cases}
$$

Next, let $s$ be any positive number and $|h|$ be sufficiently small such that $|h|<s$. After putting $v=u(s+h)$ in (1.1) for $t=s$ and $v=u(s)$ for $t=s+h$, we add these two inequalities. Then we get

$$
\begin{aligned}
& \left(D_{h} u_{t}(s), D_{h} u(s)\right)+\left\langle L_{p} u(s+h)-L_{p} u(s), D_{h} u(s)\right\rangle / h \\
& \quad \leqq\left\langle D_{h} f(s), D_{h} u(s)\right\rangle \text { a.e. } s>0
\end{aligned}
$$

where $D_{h} v(s)=(v(s+h)-v(s)) / h$. Then, Lemma 2.1 and Schwarz inequality yield that

$$
\begin{gathered}
\frac{1}{2} \frac{d}{d s}\left|D_{h} u(s)\right|_{H}^{2}+c_{0} \int_{\widehat{\Omega}}(|\nabla u(s+h)|+|\nabla u(s)|)^{p-2}\left|D_{h} \nabla u(s)\right|^{2} d x \\
\leqq\left|D_{h} f(s)\right|_{H}\left|D_{h} u(s)\right|_{H} \quad \text { a.e. } s>0
\end{gathered}
$$

where $\widehat{\Omega}=\{x \in \Omega ;|\nabla u(x, s+h)|+|\nabla u(x, s)| \neq 0\}$. By the condition of $0<p<2$, Hölder's inequality and Lemma 2.5 again

$$
\begin{gathered}
\int_{\widehat{\Omega}}\left|D_{h} \nabla u(s)\right|^{p} d x \\
=\int_{\widehat{\Omega}}\left[(|\nabla u(s+h)|+|\nabla u(s)|)^{p(p-2) / 2}\left|D_{h} \nabla u(s)\right|^{p}\right] \\
\cdot\left[(|\nabla u(s+h)|+|\nabla u(s)|)^{p(2-p) / 2}\right] d x
\end{gathered}
$$




$$
\begin{gathered}
\leqq\left(\int_{\widehat{\Omega}}(|\nabla u(s+h)|+|\nabla u(s)|)^{p-2}\left|D_{h} \nabla u(s)\right|^{2} d x\right)^{p / 2} \\
\cdot\left(\int_{\widehat{\Omega}}(|\nabla u(s+h)|+|\nabla u(s)|)^{p} d x\right)^{(2-p) / 2} \\
\leqq C\left(\int_{\widehat{\Omega}}(|\nabla u(s+h)|+|\nabla u(s)|)^{p-2}\left|D_{h} \nabla u(s)\right|^{2} d x\right)^{p / 2} .
\end{gathered}
$$

From (3.8) and (3.9) we have

$$
\begin{aligned}
& \frac{d}{d s}\left|D_{h} u(s)\right|_{H}^{2}+c\left(\int_{\widehat{\Omega}}\left|D_{h} \nabla u(s)\right|^{p} d x\right)^{2 / p} \\
& \leqq C\left|D_{h} f(s)\right|_{H}\left|D_{h} u(s)\right|_{H} \text { a.e. } s>0 .
\end{aligned}
$$

However, it is easy to see that the inequality (3.10) is valid if we replace $\widehat{\Omega}$ by $\Omega$. Then we get

$$
\frac{d}{d s}\left|D_{h} u(s)\right|_{H}^{2}+c\left|D_{h} \nabla u(s)\right|_{p}^{2} \leqq C\left|D_{h} f(s)\right|_{H}\left|D_{h} u(s)\right|_{H} \text { a.e. } s>0 .
$$

On the other hand we have by Sobolev's and Poincarè's inequalities

$$
\left|D_{h} u(s)\right|_{H} \leqq C\left|\nabla D_{h} u(s)\right|_{p}
$$

where we have used the condition of $2 n /(n+2)<p$ again. From (3.11) and (3.12) we get

$$
\frac{d}{d s}\left|D_{h} u(s)\right|_{H}^{2}+c\left|D_{h} u(s)\right|_{H}^{2} \leqq C\left|D_{h} f(s)\right|_{H}^{2} \text { a.e. } s>0 .
$$

Therefore, we obtain the following estimate from (3.13) and Lemma 2.2:

$$
\left|D_{h} u(t)\right|_{H}^{2}= \begin{cases}O\left(t^{-\beta_{1}}\right) & \text { if } \int_{t}^{t+1}\left|f_{t}(s)\right|_{H}^{2} d s=O\left(t^{-\beta_{1}}\right) \\ O\left(e^{-\nu_{2} t}\right) & \text { if } \int_{t}^{t+1}\left|f_{t}(s)\right|_{H}^{2} d s=O\left(e^{-\omega t}\right)\end{cases}
$$

with a positive constant $\nu_{2}$ for any sufficiently small $|h|<t$. In the above calculations we have used the fact that

$$
\int_{t}^{t+1}\left|D_{h} f(s)\right|_{H}^{2} d s= \begin{cases}O\left(t^{-\beta_{1}}\right) & \text { if } \int_{t}^{t+1}\left|f_{t}(s)\right|_{H}^{2} d s=O\left(t^{-\beta_{1}}\right) \\ O\left(e^{-\omega t}\right) & \text { if } \int_{t}^{t+1}\left|f_{t}(s)\right|_{H}^{2} d s=O\left(e^{-\omega t}\right)\end{cases}
$$


if $|h|$ is sufficiently small. Letting $h \rightarrow 0$ in (3.14), we have

$$
\left|u_{t}(t)\right|_{H}^{2}= \begin{cases}O\left(t^{-\beta_{1}}\right) & \text { if } \int_{t}^{t+1}\left|f_{t}(s)\right|_{H}^{2} d s=O\left(t^{-\beta_{1}}\right), \\ O\left(e^{-\nu_{2} t}\right) & \text { if } \int_{t}^{t+1}\left|f_{t}(s)\right|_{H}^{2} d s=O\left(e^{-\omega t}\right) .\end{cases}
$$

Next, we integrate $(3.8)$ over $(t, t+1)$ for any $t>0$ in order to get the following inequality from (3.14) and (3.15):

$$
\begin{aligned}
& \int_{t}^{t+1} \int_{\Omega}(|\nabla u(s+h)|+|\nabla u(s)|)^{p-2}\left|D_{h} \nabla u(s)\right|^{2} d x d s \\
& \quad \leqq C\left(\left|D_{h} u(t)\right|_{H}^{2}+\int_{t}^{t+1}\left|D_{h} f(s)\right|_{H}\left|D_{h} u(s)\right|_{H} d s\right) \\
& \quad= \begin{cases}O\left(t^{-\beta_{1}}\right) & \text { if } \int_{t}^{t+1}\left|f_{t}(s)\right|_{H}^{2} d s=O\left(t^{-\beta_{1}}\right), \\
O\left(e^{-\nu_{2} t}\right) & \text { if } \int_{t}^{t+1}\left|f_{t}(s)\right|_{H}^{2} d s=O\left(e^{-\omega t}\right) .\end{cases}
\end{aligned}
$$

Here we have defined the integrand $(|\nabla u(x, s+h)|+|\nabla u(x, s)|)^{p-2}\left|D_{h} \nabla u(x, s)\right|^{2}$ to be zero on the set $\{(x, s) \in \Omega \times(t, t+1) ;|\nabla u(x, s+h)|=|\nabla u(x, s)|=0\}$. Accordingly, by the same way as in (3.9) we obtain from (3.7) and (3.17)

$$
\begin{aligned}
\int_{t}^{t+1} \int_{\Omega}\left|D_{h} \nabla u(s)\right|^{p} d x d s & \\
= & \begin{cases}O\left(t^{-\left\{\beta_{2}+\left(\beta_{1}-\beta_{2}\right) p / 2\right\}}\right) & \text { for the case of }(\mathrm{I}), \\
O\left(e^{-\mu_{*} t}\right) & \text { for the case of }(\mathrm{II}),\end{cases}
\end{aligned}
$$

with $\mu_{*}=\min \left(\nu_{1}, \nu_{2}\right)$ for sufficiently small $|h|$. Hence, letting $h \rightarrow 0$ in (3.18), we obtain

$$
\begin{aligned}
\int_{t}^{t+1} \int_{\Omega}\left|D_{t} \nabla u\right|^{p} d x d s & \\
= & \begin{cases}O\left(t^{-\left\{\beta_{2}+\left(\beta_{1}-\beta_{2}\right) p / 2\right\}}\right) & \text { for the case of (I), } \\
O\left(e^{-\mu_{*} t}\right) & \text { for the case of (II). }\end{cases}
\end{aligned}
$$

Let us apply Lemma 2.3 to the function $z(s)=|\nabla u(s)|_{p}$ on $I=(t, t+1)$ for sufficiently large $t$. Then, for any $\varepsilon>0$,

$$
\begin{aligned}
& \sup _{t<s<t+1}|\nabla u(s)|_{p} \leqq C\left(1+\varepsilon^{-\left(p^{*}-1\right)}\right)\left\||\nabla u|_{p}\right\|_{L^{p}(t, t+1)} \\
& +\varepsilon\left\|(d / d t)|\nabla u|_{p}\right\|_{L^{p}(t, t+1)} .
\end{aligned}
$$


In (3.20) let us set $\varepsilon=t^{-\left(\beta_{2}-\beta_{1}\right) / 2 p^{*}}$ if $\beta_{1}<\beta_{2}$ and $\varepsilon=1$ if $\beta_{1}>\beta_{2}$ for the case of (I) and let us set $\varepsilon=1$ for the case of (II) again. Then, from (3.7), (3.19) and (3.20) we complete the proof of our theorem. Here we have used Lemma 2.4. In the above we have proved our theorem when $2 \leqq n$. In the case that $n=1$ the proof is given more easily.

\section{References}

[ $\mathrm{Bi}$ ] M. Biroli, Sur les inèquations paraboliques avec convexe dépendant du temps: solution forte et solution faible, Riv. Mat. Univ. Parma, 3(3) (1974), 33-72.

[ Bo ] N.M. Bokalo, Problem without initial conditions for some class of nonlinear parabolic equations, J. Soviet Math., 51(3) (1990), 2291-2322.

[C-R] M. Chipot-J.F. Rodrigues, Comparison and stability of solutions to a class of quasilinear parabolic problems, Proc. Roy. Soc. Edinburgh Sect. A, 110 (1988), 275-285.

[G-M] G.M. Ghidaglia-A. Marzocchi, Exact decay estimates for solutions to semilinear parabolic equations, Applicable Anal., 42 (1991), 69-81.

[ N1 ] H. Nagase, On an application of Rothe's method to nonlinear parabolic variational inequalities, Funkcial. Ekvac., 32-2(1989), 273-299.

[ N2 ] H. Nagase, On an asymptotic behaviour of solutions of nonlinear parabolic variational inequalities, Japan. J. Math., 15-1 (1989), 169-189.

[ T ] P. Tolksdorf, Everywhere-regularity for some quasilinear systems with lack of ellipticity, Ann. Mat. Pura Appl., 134(4) (1983), 241-266. 